\title{
EL EMPLEO VERDE EN EL ECUADOR: UN DIAGNÓSTICO NECESARIO FRENTE A LA CRISIS AMBIENTAL
}

\section{Autores:}

Raúl Cadena Palacios' \& Lida Moreno Badillo²

1. Departamento de Ciencias Humanísticas y Sociales

Universidad Tecnológica Equinoccial

Correo electrónico: dr_cadena_p@hotmail.com

Teléfono: 0985284974

Quito-Ecuador

2. Facultad de Ciencias Sociales y Comunicación Universidad Tecnológica Equinoccial

\section{REVISTA DE ․․․․ CIENTIFICA}




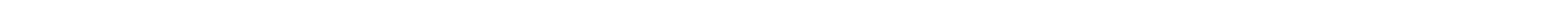




\section{RESUMEN}

Este ensayo promueve la relevancia que actualmente tiene la generación de empleo verde en el Ecuador, dentro del proceso del cambio de la matriz productiva que experimentan sectores de la economía cuyas actividades generan empleo verde como una alternativa frente a la crisis ambiental y una estrategia de desarrollo sostenible. En este contexto, se realiza un análisis cualicuantitativo de los sectores de la economía involucrados.

Palabras clave: degradación ambiental, Ecuador, desarrollo sostenible, empleo verde, economía sostenible.

\section{ABSTRACT}

This paper promotes the current relevance of generating green jobs in Ecuador, in the process of changing the productive matrix of experiencing economic sectors whose activities generate green jobs as an alternative to the environmental crisis and strategy of sustainable development. In this context, a qualitative and quantitative analysis of the economic sectors involved.

Keywords: environmental degradation, Ecuador, sustainable development, green jobs, sustainable economy. 


\section{INTRODUCCIÓN}

El presente ensayo contiene un análisis del proceso de creación de empleo verde en el Ecuador. Actualmente es implementado en algunos sectores de la economía, los cuales participan en el cambio de la matriz productiva. Esta propuesta pretende que se difundan prácticas sostenibles como un modelo innovador que ya existe en otras latitudes.

En un contexto del conflicto socio ambiental, se desprende "... una constante contradicción en las sociedades modernas. Misma que se refiere a la forma en que, en nombre del desarrollo, se echa mano de la naturaleza y sus bienes, lo cual se traduce en el deterioro y destrucción de las relaciones sociales, culturales y económicas de poblaciones humanas así como de ecosistemas enteros de una región a favor de otras que obtiene los beneficios del desarrollo" (Sasso, 2009:2). Sin embargo, no es el ánimo en esta ocasión debatir al respecto, más conviene abrir una puerta que beneficie al ser humano y a la naturaleza dentro de la nueva economía, o economía del conocimiento cuya acción en tiempo presente es la sostenibilidad. "Se entiende por economía sostenible un patrón de crecimiento que concilie el desarrollo económico, social y ambiental en una economía productiva y competitiva, que favorezca el empleo de calidad, la igualdad de oportunidades y la cohesión social (...)” (Velásquez, 2011:18). No es coincidencia que la preocupación de las sociedades actuales incorpore dentro de sus agendas, la sostenibilidad ambiental como parte del desarrollo sostenible.

Inaugurado el nuevo milenio se resalta, entre otros aspectos, el respeto a la naturaleza como valor fundamental de las relaciones internacionales y cuya meta es responsabilidad de todos los habitantes del planeta, por lo tanto se advierte que es necesario "actuar con prudencia en la gestión y ordenación de todas las especies vivas y todos los recursos naturales, conforme a los preceptos del desarrollo sostenible. Solo así podremos conservar y transmitir a nuestros descendientes las inconmensurables riquezas que nos brinda la naturaleza" (Naciones Unidas: 2000:2) de lo cual se deduce la inminente protección de nuestro entorno y el diseño de estrategias para su conservación. Aquello se ratifica en el 2003, en los Objetivos de Desarrollo del Milenio los cuales garantizarían la sostenibilidad ambiental mediante metas que permitan " incorporar los principios del desarrollo sostenible en las políticas y los programas nacionales e invertir la pérdida de recursos ambientales...reducir a la mitad, para el año 2015, la proporción de personas que carecen de acceso sostenible a agua potable...mejorar considerablemente, para el año 2020 la vida de por lo menos 100 millones de habitantes de los barrios más precarios" (PNUD, 2003:2).

En 2008 el Programa de Desarrollo de las Naciones Unidas alerta del innegable cambio climático como una amenaza grave para el desarrollo humano, reconoce la urgente necesidad de su atención y prevé que "el mundo dispone de menos de 10 años para cambiar su rumbo. No hay otro tema más urgente y ninguno exige medidas tan inmediatas como éste" (PNUD, 2008:1). Finalmente denuncia que "el cambio climático está frenando los esfuerzos por cumplir con las promesas de los ODM (Objetivos de Desarrollo del Milenio) fijados por Naciones Unidas como metas para el año 2015 Mirando hacia el futuro, el cambio climático amenaza con paralizar y revertir los avances conseguidos durante generaciones, no solo en cuanto a reducir la pobreza extrema, sino también en salud, nutrición, educación y otros ámbitos" (PNUD, 2008:2).

Dos años después, Según el organismo de Naciones Unidas (PNUD) replantea la discusión sobre el modelo de la producción y el consumo imperante frente a la tensión medioambiental y en un tono abrumador se anuncia que "...hay pocos indicios de progresos orientados a convertir el mundo en un lugar más sostenible o a proteger eficazmente de las crisis a los más vulnerables. Todavía pueden apreciarse los efectos de la crisis financiera más grande de las últimas décadas y la dependencia constante de los combustibles fósiles está amenazando con provocar daños irreparables al medio ambiente y al desarrollo humano de las generaciones venideras". (PNUD, 2010:92).

Finalmente en 2013, se plantea la crisis ambiental como el desafío apremiante del siglo XXI cuyos costos lo asumen mayormente los países pobres, (de bajos recursos) se esboza una vez más que "las amenazas ambientales, como el cambio climático, la deforestación, la contaminación del aire y el agua, y los desastres naturales, nos afectan a todos. Pero afectan más a los países y las comunidades más pobres. El cambio climático exacerba los peligros ambientales crónicos, mientras que las pérdidas de ecosistemas limitan las oportunidades de subsistencia, en especial de los más pobres" (PNUD, 2013:6).

El empleo verde entendido como aquel que reduce el impacto ambiental de las empresas y los sectores económicos hasta alcanzar niveles sostenibles, surge como una alternativa de sostenibilidad entre la economía y el medio ambiente y cuyo alcance comprende "...no solo a las actividades tradicionales de corrección y mitigación de efectos ambientales no deseados, sino otras actividades que utilizan los recursos del entorno (la biodiversidad, el suelo, la energía...) de forma que se garantice la sostenibilidad del recurso; esto es, la generación de un beneficio social y económico sin reducir dicho recurso de forma consistente" (Velásquez, 2011:19) lo cual permitiría un cambio de paradigma en la protesta social que se ajuste a una sociedad de innovación y renovación.

El cambio de la matriz productiva, planteada como uno de los grandes desafíos del gobierno actual del Ecuador con el Presidente Correa, constituye una oportunidad que permite aplicar el concepto emergente de "empleo verde". "Se han identificado 14 sectores productivos y 5 industrias estratégicas para el proceso de cambio de la matriz productiva del Ecuador. Los sectores priorizados así como las industrias estratégicas serán los que faciliten la articulación efectiva de la política pública

\section{Tsafiquiman}


y la materialización de esta transformación, pues permitirán el establecimiento de objetivos y metas específicas observables en cada una de las industrias que se intenta desarrollar" (Senplades, 2012:12). Hasta tanto, conviene identificar aquellos nuevos sectores de empleo verde consagrados en el citado modelo de producción económica que permitirán combatir la pobreza a través de la creación de plazas de empleo, la potencialización del talento humano y del conocimiento, y la reducción del daño ambiental en el marco del desarrollo sostenible; a saber los siguientes: tecnología y servicios informáticos, energías renovables, silvicultura, desarrollo agrícola, turismo, servicios ambientales etc. Según la Constitución del "Buen Vivir" en su art. 313, se denominan sectores estratégicos aquellos que por su trascendencia tienen injerencia económica, social, política o ambiental. Forman parte del sector estratégico: las telecomunicaciones, los recursos naturales no renovables, el transporte, la refinación de hidrocarburos, el patrimonio genético, el espectro radioeléctrico, el agua, y la energía en todas sus formas, entendiéndose esta última como la energía renovable en la que comprende la energía solar, eólica, biomasa y geotérmica.

"En cuanto al sector energía, se estima la creación de miles de empleos verdes en la producción de energía renovable, principalmente a partir de biomasa. De acuerdo con las estadísticas disponibles, estos empleos se han creado mayormente en China, Brasil, Estados Unidos y Alemania; sin embargo, se calcula un gran potencial en países en vías de desarrollo, lo cual permitirá a la vez mejorar la calidad de vida de personas en condiciones de pobreza al brindarles, por ejemplo, acceso a la electricidad" (Cegesti, 2012: 1), lo propio ocurriría con los otros sectores pertenecientes al nuevo modelo productivo en aras de un desarrollo sostenible.

\section{Estado actual del problema}

Con la vigencia de la actual Constitución (2008), el Estado asume la responsabilidad exclusiva de administrar, regular, controlar y gestionar los sectores estratégicos, que promueven un nuevo modelo energético en el país. Es pertinente señalar, además, que en el Ecuador desde 2003 con el apoyo del Programa de Naciones Unidas para el Desarrollo, PNUD, se lleva adelante el proyecto denominado "Ecuador: Energía Renovable para la Generación de Electricidad - Electrificación Renovable de las Islas Galápagos" ERGAL mediante la construcción de parques eólicos como es el caso de la Isla Baltra e Isla San Cristóbal, así como también, la energía fotovoltaica en la Isla Floreana e Isla Isabela, todo ello encaminado a constituirse en el 2020 como la provincia cero en combustibles fósiles.

En este sentido, actualmente en el Ecuador los "Megaproyectos" generan alrededor de 14324 empleos verdes, lo cual se presenta alentador y es propicio para fomentar e innovar el concepto emergente de empleo verde aprovechando la transformación en las relaciones de producción propuesta por el gobierno de Correa dentro de una economía diversificada así como el aprovechamiento de la capacidad, conocimiento y talento de la población ecuatoriana cuyo fin principal es el anhelado "sumak kawsay".

\section{Objetivo propuesto para la investigación}

El objetivo principal de este estudio es dar a conocer que algunos sectores de la Economía en el Ecuador, han creado actividades de empleo verde, los mismos que sirven de apoyo en la reducción o eliminación de los impactos ambientales generados por la producción contaminante, y la apertura a un trabajo digno que conciban una conciencia de cambio de actitudes frente al deterioro ambiental.

\section{Hipótesis}

La confianza en las empresas con empleo verde lleva a una mayor aceptación de las nuevas tecnologías.

\section{MÉTODO Y MATERIALES}

El presente análisis es de carácter descriptivo, social y explicativo que se sustenta en la información que se ha ido recopilando de las instituciones públicas nacionales e internacionales. Además se ha procesado la información proporcionada por las instituciones que pertenecen a los diferentes sectores económicos del país mediante estadísticas que han sido elaboradas a través de matrices y tablas explicativas.

\section{RESULTADOS Y DISCUSIÓN}

Tabla 1. Empleos verdes en el Ecuador por sectores de actividad, según el número de personas empleadas, al año 2013.

\begin{tabular}{|c|c|c|c|}
\hline $\begin{array}{l}\text { SECTORES DE ACTIVIDAD DE } \\
\text { EMPLEO VERDE }\end{array}$ & AÑo & $\begin{array}{l}\text { NUM. DE PERSONAS } \\
\text { EMPLEADAS }\end{array}$ & LUGAR \\
\hline $\begin{array}{l}\text { Proyecto Hidroeléctrico } \\
\text { COCA CODO SINCLAIR }\end{array}$ & 2013 & 4700 & $\begin{array}{c}\text { Napo (Cantón El Chaco) y } \\
\text { Sucumbíos (Cantón Gonzalo } \\
\text { Pizarro) }\end{array}$ \\
\hline $\begin{array}{l}\text { Proyecto Hidroeléctrico } \\
\text { DELSITANISAGUA }\end{array}$ & 2013 & 820 & Zamora Chinchipe \\
\hline $\begin{array}{l}\text { Proyecto Hidroeléctrico } \\
\text { MANDURIACU }\end{array}$ & 2013 & 613 & $\begin{array}{l}\text { Pacto y García Moreno (Cantón } \\
\text { Cotacachi y Quito) Pichincha e } \\
\text { Imbabura }\end{array}$ \\
\hline $\begin{array}{l}\text { Proyecto Hidroeléctrico } \\
\text { MAZAR DUDAS }\end{array}$ & 2013 & 2300 & Cañar \\
\hline Proyecto Hidroeléctrico QUIJOS & 2013 & 234 & (Cantón Quijos) Napo \\
\hline $\begin{array}{l}\text { Proyecto Hidroeléctrico } \\
\text { SOPLADORA }\end{array}$ & 2013 & 1748 & Azuay y Morona Santiago \\
\hline $\begin{array}{l}\text { Proyecto Hidroeléctrico } \\
\text { TOACHI PILATON }\end{array}$ & 2013 & 1155 & $\begin{array}{c}\text { Pichincha (Santo Domingo de } \\
\text { los Tsáchilas) }\end{array}$ \\
\hline Proyecto Eólico VILLONACO & 2013 & 254 & Loja \\
\hline REFINERIA DEL PACIFICO & 2013 & 2500 & Manabí (Cantón Manta) \\
\hline TOTAL & & 14324 & \\
\hline
\end{tabular}

Según el Ministerio de Electricidad y Energía Renovable, (Tabla №.1) el proyecto denominado "Coca Codo Sinclair" ha generado hasta el año 2013, 4700 empleos directos que han favorecido a 16559 habitantes de los cantones del Chaco en la Provincia 
de Napo, Gonzalo Pizarro en la Provincia de Sucumbíos. Este proyecto estará en la capacidad de generar $1.500 \mathrm{MW}$ de potencia efectiva con una producción energética de 8.731 GWh/ año. Es uno de los "megaproyectos" que más genera empleo verde en el Ecuador. El proyecto Hidroeléctrico "Mazar dudas" está ubicado al suroeste del Ecuador, entre las provincias del Azuay y el Cañar, específicamente en el Kilómetro 105 de la vía Cuenca-Paute-Guarumales y cuya capacidad de energía se estima en $160 \mathrm{MW}$. Este proyecto ha generado 2300 puestos de trabajos directos e indirectos, adicionalmente ha disminuido el fenómeno de la migración en estas dos provincias y la reducción de la contaminación ambiental. El proyecto Hidroeléctrico "Sopladora" está ubicado en el límite de las provincias de Azuay y Morona Santiago, con una potencia de $487 \mathrm{MW}$. El proyecto ha generado hasta el 2013, 1748 empleos directos de los cuales 953 son mano de obra calificada y 795 son mano de obra no calificada. Según el Ministerio del ramo, "Sopladora" ha generado otros beneficios a la comunidad como el empleo de bienes y servicios locales, dinamización de la economía local y mejoramientos de vías de acceso.

"Toachi Pilatón" es un proyecto que está situado en los límites de Pichincha, Santo Domingo de los Tsáchilas y la provincia de Cotopaxi, y cuya potencia es de 253 MW. Ha generado 1155 plazas de empleos verdes enmarcados en proyectos de producción agropecuaria, turismo sostenible proyecto de mejora de la captación, conducción principal y tanques de almacenamiento y potabilización del agua. Otro de los "proyectos emblemáticos" que se lleva a cabo en el país, constituye el denominado "Delsitanisagua" ubicado en el Cantón Zamora y aprovecha el potencial hidro-energético del rio Zamora. Se estima una generación de 115 MW, según el Ministerio de Electricidad y Energía Renovable, dicho proyecto beneficia especialmente a la comunidad del cantón El Chaco y sus alrededores y se emplea a alrededor de 820 personas entre mano de obra calificada y no calificada.

En este orden de creación de empleos verdes, el proyecto "Manduriacu" ha generado 613 empleos directos y según el Ministerio del ramo, también ha permitido la construcción de infraestructura educativa y centros de desarrollo infantil, áreas de salud, medicina preventiva. Este proyecto se encuentra entre las provincias de Pichincha e Imbabura y cuyo potencial energético es de $60 \mathrm{MW}$ que beneficiará a más de 2 millones de habitantes de estos dos sectores del país.

Finalmente el proyecto hidroeléctrico "Quijos" ubicado en la parroquia Cuyuja, cantón Quijos, provincia de Napo espera generar $50 \mathrm{MW}$ de potencia a nivel nacional, según el Ministerio del ramo, dicho proyecto ha generado 234 fuentes de empleo verde, pero espera alcanzar los 654 empleos directos y 1500 indirectos hasta terminar la construcción del mismo. Así mismo, se espera dinamizar la economía local mediante la contratación de gente de la localidad; mejoramiento del alumbrado público, servicios eléctricos en las viviendas y servicio de agua potable; desarrollo vial, transferencia tecnológica, entre otros beneficios. Dentro de las fuentes renovables de energía no contaminantes o limpias, se inserta el proyecto eólico "Villonaco" que permitirá desplazar la energía térmica por energía renovable y de esta manera contribuir con el cambio de la matriz productiva que actualmente experimenta el país. Dicho proyecto ha generado 254 empleos verdes, dando prioridad a la mano de obra nacional, la dinamización de la economía local, empleo de bienes y servicios locales y mejoramiento de vías de acceso. "Villonaco" se encuentra ubicado en la ciudad de Loja.

El proyecto hidrocarfurífero "Refinería del Pacífico" se desarrolla en la comunidad llamada "El Aromo" perteneciente al cantón Manta, provincia de Manabí, que busca satisfacer la demanda interna de combustibles y convertir al Ecuador en exportador de derivados. Este complejo refinador ha tenido un impacto positivo en las poblaciones aledañas generando alrededor de 2.500 puestos de empleo de los cuales el 90\% están ocupados por personas que viven en la zona de influencia del proyecto. Se espera un procesamiento de 300000 barriles diarios de crudo a partir de 2017 en el que se iniciaría oficialmente las actividades. Dentro de la Responsabilidad ambiental, este "megaproyecto" ha instalado, por ejemplo, en diferentes áreas del complejo letreros ambientales con el fin de dar a conocer el sentido de responsabilidad y protección con el medio ambiente; así por ejemplo: manejo de desechos, uso adecuado del agua, prevención de la contaminación, cuidado del ambiente, control de derrames, tiempo de degradación de desechos, etc. Se lleva adelante planes de manejo ambiental que permitan prevenir, controlar, mitigar, compensar y corregir los impactos causados por el proyecto. Entre los planes se destacan los siguientes: Plan de prevención, mitigación y compensación de impactos; plan de contingencias; plan de salud ocupacional y seguridad ambiental; plan de capacitación, que busca asegurar la integridad física de los trabajadores, contratistas, habitantes del área de influencia directa de la construcción de la vía; plan de rehabilitación de áreas afectadas.

Tabla 2. Número de empleos verdes según "proyectos emblemáticos" hidroeléctricos, eólicos e hidrocarburíferos.

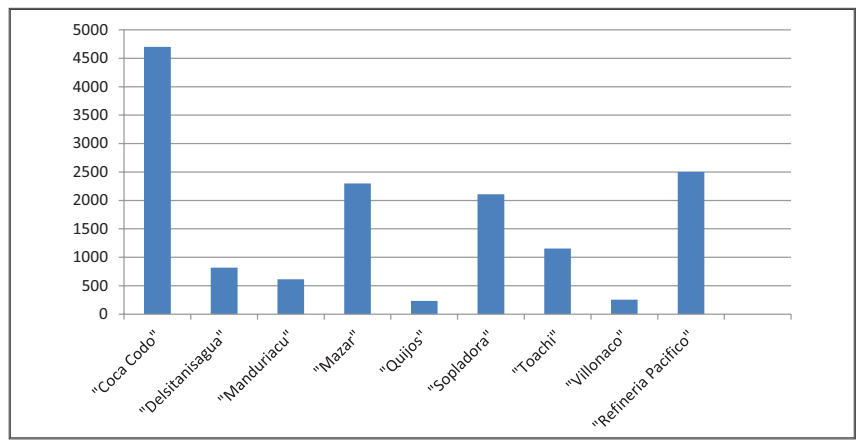

Fuente: Ministerio de Electricidad y Energía Renovable Elaboración: Raúl Cadena y Lida Moreno.

Fecha: 14 de junio de 2014

\section{TsaFiquilewax}


Al año 2013, según los datos proporcionados por el Ministerio de Electricidad y Energía Renovable, en lo referente al número de empleos verdes logrados por los "megaproyectos" hidroeléctricos, eólicos e hidrocarburíferos, encontramos la información como consta en la Tabla №.2.

Con 4700 empleos verdes se encuentra el proyecto denominado "Coca Coco Sinclair" ubicándose en el primer lugar; seguido del proyecto "Refinería del Pacifico" con 2500 empleos verdes; el proyecto "Mazar "ocupa el tercer lugar con la generación de 2 300 empleos verdes seguido de "sopladora" con 1748 empleos verdes; en quinta posición se encuentra el proyecto denominado "Toachi Pilatón" con 1155 empleos verdes, seguido de "Delsitanisagua" con 820 empleos verdes; la séptima posición la ocupa "Manduriacu" con la generación de 613 empleos verdes, seguido del proyecto eólico "Villonaco" con 254 empleos verdes; finalmente el proyecto "Quijos" ocupa la novena posición con 234 empleos verdes.

\section{CONCLUSIONES}

La crisis ambiental que soporta el planeta entero exige el diseño urgente de un nuevo modelo productivo, a través de una mejor y mayor interacción entre el hombre y los recursos naturales para lo cual se hace imperativo la concienciación y sensibilidad ambientales.

El empleo verde se constituye en una oportunidad que la propia naturaleza le concede al ser humano de remediar el daño ambiental en sectores ampliamente contaminantes como la agricultura, energía, reciclaje de residuos, transporte público, construcción, etc., además del beneficio social que deviene de éste, como la creación de nuevos yacimientos de trabajo, la mejora de la calidad de los puestos de trabajo y una mayor inclusión social.

La reducción del impacto ambiental de las empresas ecuatorianas y de los sectores económicos con la creación de los empleos verdes, constituye un aporte significativo para alcanzar niveles sostenibles.

\section{RECOMENDACIONES}

Es importante que la información existente sobre procesos productivos nuevos en los diferentes sectores de la economía del país, se dé a conocer.

Las empresas ecuatorianas y los sectores económicos, tienen una obligación moral y normativa social de crear empleos verdes, con la finalidad de alcanzar, los niveles de sostenibilidad, frente al deterioro ambiental que se produce mediante los empleos tradicionales.
La apertura a un trabajo digno que conciba una conciencia de cambio de actitudes frente al deterioro ambiental es una tarea de todos, mediante el involucramiento del sector estatal, privado y ciudadano.

\section{REFERENCIAS BIBLIOGRÁFICAS}

1. Cegesti (2012). "Empleo verde y trabajo decente". Éxito Empresarial. Disponible en: http://www.cegesti.org/ exitoempresarial/publicaciones/publicacion_193_160412_ es.pd (visitada el 02 de febrero de 2014)

2. Naciones Unidas. (2000). “Declaración del Milenio” Asamblea General de Naciones Unidas. Disponible en: http://www.un.org/ spanish/milenio/ares552.pdf (visitada el 21 de enero de 2014).

3. PNUD (2003). "Informe sobre Desarrollo Humano 2003. Los Objetivos de Desarrollo del Milenio: un pacto entre las naciones para eliminar la pobreza". Disponible en http://hdr.undp.org/ en/media/hdr03_sp_complete2.pdf (visitada el 18 de febrero de 2014).

4. PNUD (2008). "Informe sobre Desarrollo Humano 20072008. La lucha contra el cambio climático: solidaridad frente a un mundo dividido." Disponible en http://hdr.undp.org/en/ media/HDR_20072008_SP_Complete.pdf (visitada el 21 de enero de 2014).

5. PNUD (2010). "Informe sobre Desarrollo Humano 2010. La verdadera riqueza de las naciones: Caminos al desarrollo humano." Disponible en http://hdr.undp.org/en/media/ HDR_2010_ES_Complete_reprint.pdf (visitada el 21 de enero de 2014).

6. PNUD (2013). "Informe sobre Desarrollo Humano 2013. El ascenso del Sur: Progreso humano en un mundo diverso." Disponible en http://hdr.undp.org/es/informes/mundial/ idh2013/ (visitada el 21 de enero de 2014).

7. Rojas, Francisco (2012). "Seguridad Humana: nuevos enfoques". Costa Rica: FLACSO. Disponible en http://site.ebrary.com/lib/utesp/docDetail. action?docID=10592626\&p00=seguridad+ambiental (visitada el 15 de febrero de 2014).

8. Sasso, María (2009) "El proyecto multipropósito Baba: disputas sobre desarrollo y sustentabilidad. Ecuador: FLACSO. Disponible en http://ribei.org/37/3/02._Introducci\%C3\%B3n. pdf (visitada el 23 de marzo de 2014).

9. Senplades (2012). "Transformación de la Matriz Productiva. Revolución productiva a través del conocimiento y el talento humano". Secretaria Nacional de Planificación y Desarrollo

10. Velásquez, José (2011). Guía para el fomento del empleo verde en los pequeños municipios españoles. España: Rigel S.A. 
